\title{
Almost periodic solution for a Lotka-Volterra predator-prey system with feedback controls on time scales
}

\author{
Qingshui Liao ${ }^{\text {a,* }}$ \\ Guizhou Normal University, Guiyang, Guizhou, China \\ a Email:qsliao@gznu.edu.cn \\ *Qingshui Liao
}

Keywords: Lotka-Volterra system; Feedback control; Almost periodic solution; Time scales.

\begin{abstract}
By applying the theory of inequality on time scales and the Lyapunov function method, we obtain some sufficient conditions which guarantee the permanence and existence of a unique uniformly asymptotically stable almost periodic sequence solution of a Lotka-Volterra system with feedback controls.
\end{abstract}

\section{Introduction}

Recently, the dynamic behaviors of Lotka-Volterra predator-prey system have been widely investigated (e.g.see[1-3] and the references cited therein) due to their application in many fields such as physics, mechanics and the engineering technique fields. In such applications, many good results concerned with the permanence, extinction and global asymptotic stability of periodic solution or almost periodic solutions were obtained([4-6]).

However, in the previous works, one always deals with the existence of periodic solutions or almost periodic solutions to differential equations. Up to now, few works has been done for LotkaVolterra predator-prey system on time scales which can unify continuous and discrete situations. In [7], the authors propose the concept of almost periodic time scales and the definition of almost periodic functions on almost periodic time scales. Based on these, our main aim in this paper is to study the almost periodic solutions of the following Lotka-Volterra predator-prey system with feedback controls on time scales

$$
\left\{\begin{array}{l}
x_{1}^{\Delta}(t)=r_{1}(t)\left[b_{1}(t)-a_{11} \exp \left\{x_{1}(t)\right\}-a_{12} \exp \left\{x_{2}(t)\right\}+c_{1}(t) u_{1}(t)\right], \\
x_{2}^{\Delta}(t)=r_{2}(t)\left[-b_{2}(t)+a_{21} \exp \left\{x_{1}(t)\right\}-a_{22} \exp \left\{x_{2}(t)\right\}-c_{2}(t) u_{2}(t)\right], \\
u_{1}^{\Delta}(t)=f_{1}(t)-e_{1}(t) u_{1}(t)-d_{1}(t) \exp \left\{x_{1}(t)\right\}, \\
u_{2}^{\Delta}(t)=-e_{2}(t) u_{2}(t)+d_{2}(t) \exp \left\{x_{2}(t)\right\} .
\end{array}\right.
$$

where $t \in \mathbb{T}$, $\mathbb{T}$ is an almost periodic time scale, $x_{1}(t)$ is the prey population density and $x_{2}(t)$ is the predator population density. $b_{1}(t), a_{11}(t)$ the intrinsic growth rate and density-dependent coefficient of the prey, respectively; $b_{2}(t), a_{22}(t)$ the intrinsic growth rate and density-dependent coefficient of the predator, respectively; $a_{12}(t)$ the capturing rate of the predator and $a_{21}(t)$ the rate of conversion of nutrients into the reproduction of the predator. $u_{2}(t)$ and $u_{1}(t)$ are feedback controls. $f_{1}(t), r_{i}(t)$, $b_{i}(t), c_{i}(t), e_{i}(t), d_{i}(t)$ and $a_{i j}(t), i, j=1,2$ are all bounded non-negative almost periodic functions on $\mathbb{T}$.

For an almost periodic function $f: \mathbb{T} \rightarrow \mathbb{R}$, we denote $f^{M}=\sup _{t \in \mathbb{T}} f(t), f^{m}=\inf _{t \in \mathbb{T}} f(t)$, and we denote the solutions of system (1.1) by $X(t)=\left(x_{1}(t), x_{2}(t), u_{1}(t), u_{2}(t)\right)^{T}$.

Throughout this paper, we assume that $\left(\mathrm{H}_{1}\right) f_{1}(t), r_{i}(t), b_{i}(t), c_{i}(t), e_{i}(t), d_{i}(t)$ and $a_{i j}(t)$ are all bounded non-negative almost periodic functions on $\mathbb{T}$ such that

$$
\begin{aligned}
& 0<f_{1}^{m} \leq f_{1}(t) \leq f_{1}^{M}, 0<b_{i}^{m} \leq b_{i}(t) \leq b_{i}^{M}, 0<c_{i}^{m} \leq c_{i}(t) \leq c_{i}^{M}, 0<r_{i}^{m} \leq r_{i}(t) \leq r_{i}^{M}, \\
& 0<e_{i}^{m} \leq e_{i}(t) \leq e_{i}^{M}, 0<d_{i}^{m} \leq d_{i}(t) \leq d_{i}^{M}, 0<a_{i j}^{m} \leq a_{i j}(t) \leq a_{i j}^{M}, i, j=1,2 ;
\end{aligned}
$$


$\left(\mathrm{H}_{2}\right)-e_{1}^{M},-e_{1}^{m},-e_{2}^{M},-e_{2}^{m},-r_{1}^{m},-r_{2}^{m} a_{22}^{m} \in \mathcal{R}^{+}$,

$\left(\mathrm{H}_{3}\right) r_{1}^{m} b_{1}^{m}-r_{2}^{M} a_{12}^{M} \exp \left\{x_{2^{*}}\right\}>0, r_{2}^{m} a_{21}^{m} \exp \left\{x_{1^{*}}\right\}-r_{2}^{M} b_{2}^{M}-r_{2}^{M} c_{2}^{M} u_{2 *}>0$.

This paper is organized as follows: In Section 2, we introduce some notations and definitions and state some preliminary results which are needed in later sections. In Section 3, we establish some sufficient conditions for the permanence of (1.1). In Section 4, we establish some sufficient conditions for the existence of a unique almost periodic solution of (1.1).

\section{Preliminaries}

Let $\mathbb{T}$ be a nonempty closed subset (time scale) of $\mathbb{R}$. The forward and backward jump operators $\sigma, \rho: \mathbb{T} \rightarrow \mathbb{T}$ and the graininess $\mu: \mathbb{T} \rightarrow \mathbb{R}^{+}$are defined, respectively, by

$$
\sigma(t)=\inf \{s \in \mathbb{T}: s>t\}, \quad \rho(t)=\sup \{s \in \mathbb{T}: s<t\}, \quad \mu(t)=\sigma(t)-t .
$$

A point $t \in \mathbb{T}$ is called left-dense if $t>\inf \mathbb{T}$ and $\rho(t)=t$, left-scattered if $\rho(t)<t$, right-dense if $t<\sup \mathbb{T}$ and $\sigma(t)=t$, and right-scattered if $\sigma(t)>t$. If $\mathbb{T}$ has a left-scattered maximum $m$, then $\mathbb{T}^{k}=\mathbb{T} \backslash\{m\}$; otherwise $\mathbb{T}^{k}=\mathbb{T}$. If $\mathbb{T}$ has a right-scattered minimum $m$, then $\mathbb{T}_{k}=\mathbb{T} \backslash\{m\}$; otherwise $\mathbb{T}_{k}=\mathbb{T}$.

A function $f: \mathbb{T} \rightarrow \mathbb{R}$ is right-dense continuous provided it is continuous at right-dense point in $\mathbb{T}$ and its left-side limits exist at left-dense points in $\mathbb{T}$. If $f$ is continuous at each right-dense point and each left-dense point, then $f$ is said to be a continuous function on $\mathbb{T}$.

Definition 2.1[7] Assume that $f: \mathbb{T} \rightarrow \mathbb{R}$ is a function and let $t \in \mathbb{T}$. Then we define $f^{\Delta}(t)$ to be the number (provided it exists) with the property that given any $\varepsilon>0$, there is a neighborhood $U$ of $t$ (i.e., $U=(t-\delta, t+\delta) \cap \mathbb{T}$ for some $\delta>0)$ such that

$$
\left|[f(\sigma(t))-f(s)]-f^{\Delta}(t)[\sigma(t)-s]\right| \leq \varepsilon|\sigma(t)-s| \text { for all } s \in U
$$

we call $f^{\Delta}(t)$ the delta derivative of $f$ at $t$. The function $f$ is delta differentiable on $\mathbb{T}$ provided $f^{\Delta}(t)$ exists for all $t \in \mathbb{T}$. The set of functions $f: \mathbb{T} \rightarrow \mathbb{R}$ that are delta differentiable and whose delta derivative are rd-continuous functions is denoted by $C_{r d}^{1}=C_{r d}^{1}(\mathbb{T})=C_{r d}^{1}(\mathbb{T}, \mathbb{R})$.

Definition 2.2[7] A function $p: \mathbb{T} \rightarrow \mathbb{R i s}$ called regressive provided $1+\mu(t) p(t) \neq 0$ for all $t \in \mathbb{T}^{k}$. The set of all regressive and rd-continuous functions $p: \mathbb{T} \rightarrow \mathbb{R}$ will be denoted by $\mathcal{R}=\mathcal{R}(\mathbb{T})=\mathcal{R}(\mathbb{T}, \mathbb{R})$. We define the set $\mathcal{R}^{+}=\mathcal{R}^{+}(\mathbb{T}, \mathbb{R})=\{p \in \mathcal{R}: 1+\mu(t) p(t)>0, \forall t \in \mathbb{T}\}$.

Definition 2.3[7] A time scale $\mathbb{T}$ is called an almost periodic time scale if $\Pi:=\{\tau \in \mathbb{R}: t+\tau \in \mathbb{T}, \forall t \in \mathbb{T}\} \neq\{0\}$.

Throughout this paper, we restrict our discussion on almost periodic time scales.

Definition 2.4[7] Let $\mathbb{T}$ be an almost periodic time scale. A function $f: \mathbb{T} \rightarrow \mathbb{R}^{n}$ is said to be almost periodic on $\mathbb{T}$, if for any $\varepsilon>0$, the set $E(\varepsilon, f)=\{\tau \in \Pi:|f(t+\tau)-f(t)|<\varepsilon, \forall t \in \mathbb{T}\}$ is relatively dense in $\mathbb{T}$, that is, for any $\varepsilon>0$, there exists a constant $l(\varepsilon)>0$ such that each interval of length $l(\varepsilon)$ contains at least one $\tau \in E(\varepsilon, f)$ such that $|f(t+\tau)-f(t)|<\varepsilon, \forall t \in \mathbb{T}$. The set $E(\varepsilon, f)$ is called the $\varepsilon$-translation set of $f(t), \tau$ is called the $\varepsilon$-translation number of $f(t)$, and $l(\varepsilon)$ is called the inclusion of $E(\varepsilon, f)$.

Definition 2.5 System (1.1) is said to be permanent if there exist positive constants $x_{i^{*}}, x_{i}^{*}, u_{i^{*}}, u_{i}^{*}$, which are independent of the solutions of the system, such that any positive solution $X(t)$ of system (1.1) satisfies

$$
x_{i *} \leq \lim \inf _{t \rightarrow+\infty} x_{i}(t) \leq \lim \sup _{t \rightarrow+\infty} x_{i}(t) \leq x_{i}^{*}, \quad u_{i \star} \leq \lim \inf _{t \rightarrow+\infty} u_{i}(t) \leq \lim \sup _{t \rightarrow+\infty} u_{i}(t) \leq u_{i}^{*}, \quad i=1,2 .
$$

Lemma 2.1[7] Let $-a \in \mathcal{R}^{+}$.

(i) If $x^{\Delta}(t) \leq b-a x(t)$, then for $t>t_{0}, x(t) \leq x\left(t_{0}\right) e_{(-a)}\left(t, t_{0}\right)+\frac{b}{a}\left(1-e_{(-a)}\left(t, t_{0}\right)\right)$. In particular, if $a>0$, we 
have $\limsup _{t \rightarrow+\infty} x(t) \leq \frac{b}{a}$.

(ii) If $x^{\Delta}(t) \geq b-a x(t)$, then for $t>t_{0}, x(t) \geq x\left(t_{0}\right) e_{(-a)}\left(t, t_{0}\right)+\frac{b}{a}\left(1-e_{(-a)}\left(t, t_{0}\right)\right)$. In particular, if $a>0$, we have $\liminf _{t \rightarrow+\infty} x(t) \geq \frac{b}{a}$.

Lemma 2.2[7] Suppose that there exists a Lyapunov function $V(t, x, y)$ satisfying the following conditions

(i) $a(|x-y|) \leq V(t, x, y) \leq b(|x-y|)$, where $a, b \in \mathcal{K}, \mathcal{K}=\left\{a \in C\left(R^{+}, R^{+}\right): a(0)=0\right.$ and $a(x)$ is increasing in $x\}$

(ii) $\left|V\left(t, x_{1}, y_{1}\right)-V\left(t, x_{2}, y_{2}\right)\right| \leq L\left(\left|x_{1}-x_{2}\right|+\left|y_{1}-y_{2}\right|\right)$, where $L>0$ is a constant;

(iii) $D^{+} V^{\Delta}(t, x, y) \leq-c V(t, x, y)$ where $-c \in \mathcal{R}^{+}$, and $c>0$.

Moreover, if there exists a solution $x(t)$ of $x^{\Delta}=f(t, x)$ such that $x(t) \in \mathbb{S}$ for all $t \in \mathbb{T}^{+}$, where $S \subset D$ is a compact set. Then there exists a unique uniformly asymptotically stable almost periodic solution $p(t)$ in $S$.

\section{Permanence}

In this section, we establish some permanence results for system (1.1). From Lemma 2.1, one can obtain the following results.

Theorem 3.1 Assume that $\left(\mathrm{H}_{1}\right),\left(\mathrm{H}_{2}\right)$ hold. Then every solution $X(t)$ of system (1.1) satisfies

Where

$$
\lim \sup _{t \rightarrow+\infty} x_{1}(t) \leq x_{1}^{*}, \lim \sup _{t \rightarrow+\infty} x_{2}(t) \leq x_{2}^{*}, \lim \sup _{t \rightarrow+\infty} u_{1}(t) \leq u_{1}^{*}, \lim \sup _{t \rightarrow+\infty} u_{1}(t) \leq u_{2}^{*} \text {, }
$$

$$
x_{1}^{*}=\frac{r_{1}^{M} b_{1}^{M}+r_{1}^{M} c_{1}^{M} u_{1}^{*}-r_{1}^{m} a_{11}^{m}}{r_{1}^{m} a_{11}^{m}}, x_{2}^{*}=\frac{r_{2}^{M} a_{21}^{M} \exp \left\{x_{1}^{*}\right\}-r_{2}^{m} a_{22}^{m}}{r_{2}^{m} a_{22}^{m}}, u_{1}^{*}=\frac{f_{1}^{M}}{e_{1}^{m}}, u_{2}^{*}=\frac{d_{2}^{M} \exp \left\{x_{2}^{*}\right\}}{e_{2}^{m}} .
$$

Proof From the third equation of (1.1), we have

$$
u_{1}^{\Delta}(t)=f_{1}(t)-e_{1}(e) u_{1}(t)-d_{1}(t) \exp \left\{x_{1}(t)\right\} \leq f_{1}^{M}-e_{1}^{m} u_{1}(t) .
$$

It follows from the condition (i) of Lemma 2.1

$$
\lim \sup _{t \rightarrow+\infty} u_{1}(t) \leq u_{1}^{*}
$$

the first equation of (1.1) we have

$$
\begin{aligned}
x_{1}^{\Delta}(t) & =r_{1}(t)\left[b_{1}(t)-a_{11}(t) \exp \left\{x_{1}(t)\right\}-a_{12}(t) \exp \left\{x_{2}(t)\right\}+c_{1}(t) u_{1}(t)\right] \\
& \leq r_{1}^{M} b_{1}^{M}+r_{1}^{M} c_{1}^{M} u_{1}^{*}-r_{1}^{m} a_{11}^{m}-r_{1}^{m} a_{11}^{m} x_{1}(t),
\end{aligned}
$$

follows from Lemma 2.1(i), that

$$
\lim \sup _{t \rightarrow+\infty} x_{1}(t) \leq x_{1}^{*} .
$$

Then for any $\varepsilon>0$, there exists a $t_{0} \in \mathbb{T}$ such that $x_{1}(t) \leq x_{1}^{*}+\varepsilon$ for all $t \geq t_{0}$.

While, from the second equation of (1.1), we get

$$
\begin{aligned}
x_{2}^{\Delta}(t) & =r_{2}(t)\left[-b_{2}(t)+a_{21}(t) \exp \left\{x_{1}(t)\right\}-a_{22}(t) \exp \left\{x_{2}(t)\right\}-c_{2}(t) u_{2}(t)\right] \\
& \leq r_{2}^{M} a_{21}^{M} \exp \left\{x_{1}^{*}+\varepsilon\right\}-r_{2}^{m} a_{22}^{m}-r_{2}^{m} a_{22}^{m} x_{2}(t) .
\end{aligned}
$$

Letting $\varepsilon \rightarrow 0$, we get $\lim \sup x_{2}(t) \leq x_{2}^{*}$. It follows from Lemma 2.1(i), that

$$
\lim \sup _{t \rightarrow+\infty} x_{2}(t) \leq x_{2}^{*} .
$$

From the last equation of (1.1), we arrive

$$
u_{2}^{\Delta}(t)=-e_{2}(t) u_{2}(t)+d_{2}(t) \exp \left\{x_{2}(t)\right\} \leq d_{2}^{M} \exp \left\{x_{2}^{*}+\varepsilon\right\}-e_{2}^{m} u_{2}(t),
$$

then we have

$$
\limsup u_{2}(t) \leq u_{2}^{*} \text {. }
$$


Similarly, we can get the following theorem.

Theorem 3.2 Assume that $\left(\mathrm{H}_{1}\right)-\left(\mathrm{H}_{3}\right)$ hold, then every solution $X(t)$ of system (1.1) satisfies $\lim \inf _{t \rightarrow+\infty} x_{1}(t) \geq x_{1,}, \lim \inf _{t \rightarrow+\infty} x_{2}(t) \geq x_{2 *}, \lim \inf _{t \rightarrow+\infty} u_{1}(t) \geq u_{1,}, \lim \inf _{t \rightarrow+\infty} u_{2}(t) \geq u_{2 *}$,

where

$$
\begin{aligned}
& x_{1_{*}}=\ln \frac{r_{1}^{m} b_{1}^{m}-r_{1}^{M} a_{12}^{M} \exp \left\{x_{2}^{*}\right\}}{r_{1}^{M} a_{11}^{M}}, u_{1_{*}}=\frac{f_{1}^{m}-d_{1}^{M} \exp \left\{x_{1}^{*}\right\}}{e_{1}^{M}}, \\
& x_{2_{*}}=\ln \frac{-r_{2}^{M} b_{2}^{M}+r_{2}^{m} a_{21}^{m} \exp \left\{x_{1_{*}}\right\}-r_{2}^{M} c_{2}^{M} u_{2}^{*}}{r_{2}^{M} a_{22}^{M}}, u_{2 *}=\frac{d_{2}^{m} \exp \left\{x_{2 *}\right\}}{e_{2}^{M}} .
\end{aligned}
$$

Theorem 3.3 Assume that $\left(\mathrm{H}_{1}\right)-\left(\mathrm{H}_{3}\right)$ hold. Then system (1.1) is permanent.

\section{Existence of a unique almost periodic solution}

We denote the set of all the solutions $X(t)=\left(x_{1}(t), x_{2}(t), u_{1}(t), u_{2}(t)\right)$ of system (1.1) which satisfyin $x_{1 *} \leq x_{1}(t) \leq x_{1}^{*}, x_{2 *} \leq x_{2}(t) \leq x_{2}^{*}, u_{1 *} \leq u_{1}(t) \leq u_{1}^{*}, u_{2 *} \leq u_{2}(t) \leq u_{2}^{*}, t \in \mathbb{T}$ by $\Omega$. According to Theorem 3.3, we have the following result.

Theorem 4.1 Assume that $\left(\mathrm{H}_{1}\right)-\left(\mathrm{H}_{3}\right)$ hold. Then $\Omega \neq \varnothing$.

Theorem 4.2 Assume that $\left(\mathrm{H}_{1}\right)-\left(\mathrm{H}_{3}\right)$ hold. And furthermore assume that $\left(\mathrm{H}_{4}\right) \Theta>0$ and $-\Theta \in \mathcal{R}^{+}$, where $\Theta=\min \{E, F, G, H\}$, and

$$
\begin{aligned}
E= & d_{1}^{m} \exp \left\{\xi_{1^{*}}\right\}-r_{1}^{M} c_{1}^{M}-r_{2}^{M} a_{12}^{M} \exp \left\{\xi_{1}^{*}\right\}-\mu^{M} d_{1}^{M} \exp \left\{\xi_{1}^{*}\right\}\left[e_{1}^{M}+d_{1}^{M} \exp \left\{\xi_{1}^{*}\right\}\right] \\
& -\mu^{M} r_{2}^{2 M} a_{21}^{2 M} \exp \left\{2 \xi_{2}^{*}\right\}-\mu^{M} r_{1}^{2 M} a_{11}^{M} \exp \left\{\xi_{1}^{*}\right\}\left[a_{12}^{M} \exp \left\{\xi_{2}^{*}\right\}+a_{11}^{M} \exp \left\{\xi_{1}^{*}\right\}\right], \\
F= & r_{1}^{m} a_{12}^{m} \exp \left\{\xi_{2_{*}}\right\}-d_{2}^{M} \exp \left\{\xi_{2}^{*}\right\}-r_{2}^{M} a_{21}^{M} \exp \left\{\xi_{1}^{*}\right\}-\mu^{M} r_{2}^{2 M} a_{22}^{M} \exp \left\{\xi_{2}^{*}\right\}\left[c_{2}^{M}+a_{22}^{M} \exp \left\{\xi_{2}^{*}\right\}\right] \\
& -\mu^{M} d_{2}^{2 M} \exp \left\{2 \xi_{2}^{*}\right\}-\mu^{M} r_{1}^{2 M} a_{12}^{M} \exp \left\{\xi_{2}^{*}\right\}\left[a_{11}^{M} \exp \left\{\xi_{1}^{*}\right\}+a_{12}^{M} \exp \left\{\xi_{2}^{*}\right\}\right], \\
G= & d_{1}^{m} \exp \left\{\xi_{1^{*}}\right\}-r_{1}^{M} c_{1}^{M}-\mu^{M} r_{1}^{2 M} c_{1}^{2 M}-\mu^{M} e_{1}^{M}\left[e_{1}^{M}+d_{1}^{M} \exp \left\{\xi_{1}^{*}\right\}\right], \\
H= & 2 e_{2}^{m}-d_{2}^{M} \exp \left\{\xi_{2}^{*}\right\}-\mu^{M} e_{2}^{2 M}-\mu^{M} r_{2}^{2 M} c_{2}^{M}\left[c_{2}^{M}+a_{22}^{M} \exp \left\{\xi_{2}^{*}\right\}\right] .
\end{aligned}
$$

Then there exists a unique uniformly asymptotically stable almost periodic solution $X(t)$ of system (1.1), and $X(t) \in \Omega$.

Proof From Theorem 4.1, there exists $X(t)$ such that

$$
x_{1 *} \leq x_{1}(t) \leq x_{1}^{*}, x_{2 *} \leq x_{2}(t) \leq x_{2}^{*}, u_{1 *} \leq u_{1}(t) \leq u_{1}^{*}, u_{2 *} \leq u_{2}(t) \leq u_{2}^{*}, t \in \mathbb{T} .
$$

We denote

then,

$$
A_{1}=\max \left\{\left|x_{1^{*}}\right|,\left|x_{1}^{*}\right|\right\}, A_{2}=\max \left\{\left|x_{2^{*}}\right|,\left|x_{2}^{*}\right|\right\}, A_{3}=\max \left\{\left|u_{1^{*}}\right|,\left|u_{1}^{*}\right|\right\}, A_{4}=\max \left\{\left|u_{2^{*}}\right|,\left|u_{2}^{*}\right|\right\} .
$$

Define the norm

$$
\left|x_{1}(t)\right| \leq A_{1},\left|x_{2}(t)\right| \leq A_{2},\left|u_{1}(t)\right| \leq A_{3},\left|u_{2}(t)\right| \leq A_{4} .
$$

$$
\| X\left|=\sup _{t \in \mathbb{T}}\right| x_{1}(t)\left|+\sup _{t \in \mathbb{T}}\right| x_{2}(t)\left|+\sup _{t \in \mathbb{T}}\right| u_{1}(t)\left|+\sup _{t \in \mathbb{T}}\right| u_{2}(t) \mid, X(t) \in \mathbb{R}^{4} .
$$

In view of Theorem 4.1, we can suppose that

$$
X_{1}=\left(x_{1}(t), x_{2}(t), u_{1}(t), u_{2}(t)\right)^{T}, Y_{1}=\left(m_{1}(t), m_{2}(t), n_{1}(t), n_{2}(t)\right)^{T},
$$

be any two positive solutions of system (1.1).

Substituting (4.2) into the system of (1.1), we arrive to

$$
\left\{\begin{aligned}
\left(x_{1}-m_{1}\right)^{\Delta}(t)= & r_{1}(t)\left[-a_{11}(t)\left[\exp \left\{x_{1}(t)\right\}-\exp \left\{m_{1}(t)\right\}\right]\right. \\
& \left.-a_{12}(t)\left(\exp \left\{x_{2}(t)\right\}-\exp \left\{m_{2}(t)\right\}\right)+c_{1}(t)\left(u_{1}(t)-n_{1}(t)\right)\right] \\
\left(x_{2}-m_{2}\right)^{\Delta}(t)= & r_{2}(t)\left[a_{21}(t)\left(\exp \left\{x_{1}(t)\right\}-\exp \left\{m_{1}(t)\right\}\right)\right. \\
& \left.-a_{22}(t)\left[\exp \left\{x_{2}(t)\right\}-\exp \left\{m_{2}(t)\right\}\right]-c_{2}(t)\left[u_{2}(t)-n_{2}(t)\right]\right] \\
\left(u_{1}-n_{1}\right)^{\Delta}(t)= & -e_{1}(t)\left[u_{2}(t)-n_{2}(t)\right]-d_{1}(t)\left[\exp \left\{x_{1}(t)\right\}-\exp \left\{m_{1}(t)\right\}\right] \\
\left(u_{2}-n_{2}\right)^{\Delta}(t)= & -e_{2}(t)\left[u_{2}(t)-n_{2}(t)\right]+d_{2}(t)\left[\exp \left\{x_{2}(t)\right\}-\exp \left\{m_{2}(t)\right\}\right]
\end{aligned}\right.
$$


Considering Lyapunov function $V\left(t, X_{1}, Y_{1}\right)$ on $\mathbb{T}^{+} \times \Omega \times \Omega$ defined by

$$
V\left(t, X_{1}, Y_{1}\right)=\left(x_{1}(t)-m_{1}(t)\right)^{2}+\left(x_{2}(t)-m_{2}(t)\right)^{2}+\left(u_{1}(t)-n_{1}(t)\right)^{2}+\left(u_{2}(t)-n_{2}(t)\right)^{2} .
$$

Obviously, the norm of (4.1) is equated with the following form of norm

$$
\|X\|_{*}=\sup _{t \in \mathbb{T}}\left[\left(x_{1}(t)\right)^{2}+\left(x_{2}(t)\right)^{2}+\left(u_{1}(t)\right)^{2}+\left(u_{2}(t)\right)^{2}\right]^{\frac{1}{2}} .
$$

Then, $\|\not\|_{1}-Y_{1 *}$ and $\| X_{1}-Y_{1}$ are equivalent. Then, there exist two constants $C_{1}>0, C_{2}>0$ such that

$$
C_{1}\left\|X_{1}+Y Y_{\|} \sharp\right\| X_{1}-Y_{1 *} \leq C_{2} \quad X_{1}-Y_{1}
$$

Take $a, b \in C\left(\mathbb{R}^{\star}, \mathbb{R}^{\dagger}\right), a(x)=C_{1}^{2} x^{2}, b(x)=C_{2}^{2} x^{2}$, thus the condition (i) of Lemma 2.2 is satisfied. In addition,

$$
\begin{aligned}
\left|V\left(t, X_{1}, Y_{1}\right)-V\left(t, X_{2}, Y_{2}\right)\right|= & \mid\left[\left(x_{1}(t)-m_{1}(t)\right)^{2}+\left(x_{2}(t)-m_{2}(t)\right)^{2}+\left(u_{1}(t)-n_{1}(t)\right)^{2}+\left(u_{2}(t)-n_{3}(t)\right)^{2}\right] \\
& -\left[\left(x_{1}^{\prime}(t)-m_{1}^{\prime}(t)\right)^{2}+\left(x_{2}^{\prime}(t)-m_{2}^{\prime}(t)\right)^{2}+\left(u_{1}^{\prime}(t)-n_{1}^{\prime}(t)\right)^{2}+\left(u_{2}^{\prime}(t)-n_{3}^{\prime}(t)\right)^{2}\right] \mid \\
\leq & L\left[\left(\left|x_{1}(t)-x_{1}^{\prime}(t)\right|+\left|m_{1}(t)-m_{1}^{\prime}(t)\right|\right)+\left(\left|x_{2}(t)-x_{2}^{\prime}(t)\right|+\left|m_{2}(t)-m_{2}^{\prime}(t)\right|\right)\right. \\
& \left.+\left(\left|u_{1}(t)-u_{1}^{\prime}(t)\right|+\left|n_{1}(t)-n_{1}^{\prime}(t)\right|\right)+\left(\left|u_{2}(t)-u_{2}^{\prime}(t)\right|+\left|n_{2}(t)-n_{2}^{\prime}(t)\right|\right)\right] \\
\leq & L\left(\|\left. X_{1} H X\right|_{2}+Y_{1}-Y_{2}\right) .
\end{aligned}
$$

where

$$
\begin{aligned}
& L=4 \max _{i=1,2,3,4} A_{i}, \\
& X_{2}=\left(x_{1}^{\prime}(t), x_{2}^{\prime}(t), \ldots, x_{N}^{\prime}\left((t), u_{1}^{\prime}(t), u_{2}^{\prime}(t), \ldots, u_{N}^{\prime}(t)\right)^{T},\right. \\
& Y_{2}=\left(m_{1}^{\prime}(t), m_{2}^{\prime}(t), \ldots, m_{N}^{\prime}(t), n_{1}(t), n_{2}^{\prime}(t), \ldots, n_{N}^{\prime}(t)\right)^{T} .
\end{aligned}
$$

Thus the condition (ii) of Lemma 2.2 is also satisfied. Finally, we will prove the condition (iii) of Lemma 2.2 is satisfied, calculating the right derivative $D^{+} V^{\Delta}$ of $V$ along the solution of (4.4).

$$
\begin{aligned}
D^{+} V^{\Delta}\left(t, X_{1}, Y_{1}\right)= & {\left[2\left(x_{1}(t)-m_{1}(t)\right)+\mu(t)\left(x_{1}(t)-m_{1}(t)\right)^{\Delta}\right]\left(x_{1}(t)-m_{1}(t)\right)^{\Delta} } \\
& +\left[2\left(x_{2}(t)-m_{2}(t)\right)+\mu(t)\left(x_{2}(t)-m_{2}(t)\right)^{\Delta}\right]\left(x_{2}(t)-m_{2}(t)\right)^{\Delta} \\
& +\left[2\left(u_{1}(t)-n_{1}(t)\right)+\mu(t)\left(u_{1}(t)-n_{1}(t)\right)^{\Delta}\right]\left(u_{1}(t)-n_{1}(t)\right)^{\Delta} \\
& +\left[2\left(u_{2}(t)-n_{2}(t)\right)+\mu(t)\left(u_{2}(t)-n_{2}(t)\right)^{\Delta}\right]\left(u_{2}(t)-n_{2}(t)\right)^{\Delta} \\
= & V_{1}+V_{2}+V_{3}+V_{4},
\end{aligned}
$$

where

$$
\begin{aligned}
& V_{1}=\left[2\left(x_{1}(t)-m_{1}(t)\right)+\mu(t)\left(x_{1}(t)-m_{1}(t)\right)^{\Delta}\right]\left(x_{1}(t)-m_{1}(t)\right)^{\Delta}, \\
& V_{2}=\left[2\left(x_{2}(t)-m_{2}(t)\right)+\mu(t)\left(x_{2}(t)-m_{2}(t)\right)^{\Delta}\right]\left(x_{2}(t)-m_{2}(t)\right)^{\Delta}, \\
& V_{3}=\left[2\left(u_{1}(t)-n_{1}(t)\right)+\mu(t)\left(u_{1}(t)-n_{1}(t)\right)^{\Delta}\right]\left(u_{1}(t)-n_{1}(t)\right)^{\Delta}, \\
& V_{4}=\left[2\left(u_{2}(t)-n_{2}(t)\right)+\mu(t)\left(u_{2}(t)-n_{2}(t)\right)^{\Delta}\right]\left(u_{2}(t)-n_{2}(t)\right)^{\Delta} .
\end{aligned}
$$

By the mean value theorem, we get

$\exp \left\{x_{1}(t)\right\}-\exp \left\{m_{1}(t)\right\}=\exp \left\{\xi_{1}(t)\right\}\left(x_{1}(t)-m_{1}(t)\right), \exp \left\{x_{2}(t)\right\}-\exp \left\{m_{2}(t)\right\}=\exp \left\{\xi_{2}(t)\right\}\left(x_{2}(t)-m_{2}(t)\right)$, where $\xi_{1}(t), \xi_{2}(t)$ lie between $x_{1}(t)$ and $m_{1}(t), x_{2}(t)$ and $m_{2}(t)$ respectively. Then, (4.3) can be rewritten as

$$
\left\{\begin{array}{l}
\left(x_{1}-m_{1}\right)^{\Delta}(t)=-r_{1}(t)\left[a_{11}(t) \exp \left\{\xi_{1}(t)\right\}\left(x_{1}(t)-m_{1}(t)\right)+a_{12}(t) \exp \left\{\xi_{2}(t)\right\}\left(x_{2}(t)-m_{2}(t)\right)-c_{1}(t)\left(u_{1}(t)-n_{1}(t)\right)\right], \\
\left(x_{2}-m_{2}\right)^{\Delta}(t)=-r_{2}(t)\left[a_{22}(t) \exp \left\{\xi_{2}(t)\right\}\left(x_{2}(t)-m_{2}(t)\right)-a_{21}(t) \exp \left\{\xi_{1}(t)\right\}\left(x_{1}(t)-m_{1}(t)\right)+c_{2}(t)\left(u_{2}(t)-n_{2}(t)\right)\right], \\
\left(u_{1}-n_{1}\right)^{\Delta}(t)=-e_{1}(t)\left(u_{1}(t)-n_{1}(t)\right)-d_{1}(t) \exp \left\{\xi_{1}(t)\right\}\left(x_{1}(t)-m_{1}(t)\right), \\
\left(u_{2}-n_{2}\right)^{\Delta}(t)=-e_{2}(t)\left(u_{2}(t)-n_{2}(t)\right)+d_{2}(t) \exp \left\{\xi_{2}(t)\right\}\left(x_{2}(t)-m_{2}(t)\right) .
\end{array}\right.
$$

According to Theorem 4.2 and (4.6), and combing with the inequality of $2|a \| b| \leq a^{2}+a^{2}$ then we have 


$$
\begin{aligned}
& V_{1}=-r_{1}(t)\left(2\left(x_{1}(t)-m_{1}(t)\right)-\mu(t) r_{1}(t)\left[a_{11}(t) \exp \left\{\xi_{1}(t)\right\}\left(x_{1}(t)-m_{1}(t)\right)\right.\right. \\
& \left.\left.\left.+a_{12}(t) \exp \left\{\xi_{2}(t)\right\}\left(x_{2}(t)-m_{2}(t)\right)-c_{1}(t)\left(u_{1}(t)-n_{1}(t)\right)\right]\right\}\right) \\
& \times\left[a_{11}(t) \exp \left\{\xi_{1}(t)\right\}\left(x_{1}(t)-m_{1}(t)\right)-c_{1}(t)\left(u_{1}(t)-n_{1}(t)\right)+a_{12}(t) \exp \left\{\xi_{2}(t)\right\}\left(x_{2}(t)-m_{2}(t)\right)\right] \\
& \leq\left[a_{12}^{M} \exp \left\{\xi_{2}^{*}\right\}-r_{1}^{m} a_{12}^{m} \exp \left\{\xi_{2 *}\right\}+\mu^{M} r_{1}^{2 M} a_{12}^{M} \exp \left\{\xi_{2}^{*}\right\}\left(a_{11}^{M} \exp \left\{\xi_{1}^{*}\right\}\right)\right]\left(x_{2}(t)-m_{2}(t)\right)^{2} \\
& +\left[r_{1}^{M} c_{1}^{M}+\mu^{M} r_{1}^{2 M} a_{11}^{M} \exp \left\{\xi_{1}^{*}\right\}\left(a_{12}^{M} \exp \left\{\xi_{2}^{*}\right\}+a_{11}^{M} \exp \left\{\xi_{1}^{*}\right\}\right)\right]\left(x_{1}(t)-m_{1}(t)\right)^{2} \\
& +\left(r_{1}^{M} c_{1}^{M}+\mu^{M} r_{1}^{2 M} c_{1}^{2 M}\right)\left(u_{1}(t)-n_{1}(t)\right)^{2}, \\
& V_{2}=-r_{2}(t)\left(2\left(x_{2}(t)-m_{2}(t)\right)-\mu(t) r_{2}(t)\left[a_{22}(t) \exp \left\{\xi_{2}(t)\right\}\left(x_{2}(t)-m_{2}(t)\right)\right.\right. \\
& \left.\left.-a_{21}(t) \exp \left\{\xi_{1}(t)\right\}\left(x_{1}(t)-m_{1}(t)\right)+c_{2}(t)\left(u_{2}(t)-n_{2}(t)\right)\right]\right) \\
& \times\left[a_{22}(t) \exp \left\{\xi_{2}(t)\right\}\left(x_{2}(t)-m_{2}(t)\right)+c_{2}(t)\left(u_{2}(t)-n_{2}(t)\right)-a_{21}(t) \exp \left\{\xi_{1}(t)\right\}\left(x_{1}(t)-m_{1}(t)\right)\right] \\
& \leq\left[r_{2}^{M} a_{21}^{M} \exp \left\{\xi_{1}^{*}\right\}+\mu^{M} r_{2}^{2 M} a_{22}^{M} \exp \left\{\xi_{2}^{*}\right\}\left(c_{2}^{M}+a_{22}^{M} \exp \left\{\xi_{2}^{*}\right\}\right)\right]\left(x_{2}(t)-m_{2}(t)\right)^{2} \\
& +\left(r_{2}^{M} a_{12}^{M} \exp \left\{\xi_{1}^{*}\right\}+\mu^{M} r_{2}^{2 M} a_{21}^{2 M} \exp \left\{2 \xi_{2}^{*}\right\}\right)\left(x_{1}(t)-m_{1}(t)\right)^{2} \\
& +\mu^{M} r_{2}^{2 M} c_{2}^{M}\left(c_{2}^{M}+a_{22}^{M} \exp \left\{\xi_{2}^{*}\right\}\right)\left(u_{2}(t)-n_{2}(t)\right)^{2}, \\
& V_{3}=-\left(2\left(u_{1}(t)-n_{1}(t)\right)-\mu(t)\left[e_{1}(t)\left(u_{1}(t)-n_{1}(t)\right)+d_{1}(t) \exp \left\{\xi_{1}(t)\right\}\left(x_{1}(t)-m_{1}(t)\right)\right]\right) \\
& \times\left[e_{1}(t)\left(u_{1}(t)-n_{1}(t)\right)+d_{1}(t) \exp \left\{\xi_{1}(t)\right\}\left(x_{1}(t)-m_{1}(t)\right)\right] \\
& \leq\left[-d_{1}^{m} \exp \left\{\xi_{1_{*}}\right\}+\mu^{M} d_{1}^{M} \exp \left\{\xi_{1}^{*}\right\}\left(e_{1}^{M}+d_{1}^{M} \exp \left\{\xi_{1}^{*}\right\}\right)\right]\left(x_{1}(t)-m_{1}(t)\right)^{2} \\
& +\left[-d_{1}^{m} \exp \left\{\xi_{1_{*}}\right\}+\mu^{M} e_{1}^{M}\left(e_{1}^{M}+d_{1}^{M} \exp \left\{\xi_{1}^{*}\right\}\right)\right]\left(u_{1}(t)-n_{1}(t)\right)^{2}, \\
& V_{4}=-\left(2\left(u_{2}(t)-n_{2}(t)\right)-\mu(t)\left[e_{2}(t)\left(u_{2}(t)-n_{2}(t)\right)-d_{2}(t) \exp \left\{\xi_{2}(t)\right\}\left(x_{2}(t)-m_{2}(t)\right)\right]\right) \\
& \times\left[e_{2}(t)\left(u_{2}(t)-n_{2}(t)\right)-d_{2}(t) \exp \left\{\xi_{2}(t)\right\}\left(x_{2}(t)-m_{2}(t)\right)\right] \\
& \leq\left[-2 e_{2}^{m}+d_{2}^{M} \exp \left\{\xi_{2}^{*}\right\}+\mu^{M} e_{2}^{2 M}\right]\left(u_{2}(t)-n_{2}(t)\right)^{2} \\
& +\left(d_{2}^{M} \exp \left\{\xi_{2}^{*}\right\}+\mu^{M} d_{2}^{2 M} \exp \left\{2 \xi_{2}^{*}\right\}\right)\left(x_{2}(t)-m_{2}(t)\right)^{2} .
\end{aligned}
$$

In view of (4.7) - (4.10), we obtain

$$
D^{+} V^{\Delta}\left(t, X_{1}, U_{1}\right)=V_{1}+V_{2}+V_{3}+V_{4} \leq-\Theta V\left(t, X_{1}, Y_{1}\right) .
$$

Therefore, condition $\left(\mathrm{H}_{4}\right)$ is satisfied. Hence, according to Lemma 2.2, there exists a unique uniformly asymptotically stable almost periodic solution $X(t)$ of system (1.1), and $X(t) \in \Omega$.

\section{References}

[1] Faria, T., Muroya, Y. (2015) Global attractivity and extinction for Lotka-Volterra systems with infinite delay and feedback controls. P. Roy. Soc. Edinb. A., 145(2), 301-330.

[2] Yang, J., Tang, S. (2016) Holling type II predator-prey model with nonlinear pulse as statedependent feedback control. J. Comput. Appl. Math., 291, 225-241.

[3] Liao, Q.S., Li, B., Li, Y. K. (2015) Permanence and almost periodic solutions for an n-species Lotkau-Volterra food chain system on time scales. Asian-European J. Math., 8(02), 1152-1160.

[4] Huang, Z., Chen, F. (2005) Almost periodic solution in two species competitive system with feedback controls. J. Biomath., 20(1), 28-32.

[5] Geng, J.B., Xia, Y.H. (2011) Almost periodic solutions of a nonlinear ecological model. Commun. Nonlinear Sci., 16, 2575-2597.

[6] Liu, Q., Jiang, D., Hayat, T., et al. (2018) Dynamics of a Stochastic Predator-Prey Model with Stage Structure for Predator and Holling Type II Functional Response. J. Nonlinear Sci., 1-37.

[7] Li, Y.K., Wang, C. (2011) Uniformly Almost Periodic Functions and Almost Periodic Solutions to Dynamic Equations on Time Scales. Abstract and Applied Analysis, Article ID 341520, 23 pages. 